\title{
Alain Mothu, Gianluca Mori (textes réunis par), Philosophes sans Dieu.Textes athées clandestins du XVIII ${ }^{\mathrm{e}}$ siècle \\ Paris, Ếditions Honoré Champion-Classiques, [2005] 2010, 400 p. \\ Daniel Vidal
}

\section{(2) OpenEdition}

\section{Journals}

Édition électronique

URL : http://journals.openedition.org/assr/22930

DOI : 10.4000/assr.22930

ISSN : $1777-5825$

Éditeur

Éditions de l'EHESS

Édition imprimée

Date de publication : 31 décembre 2011

Pagination : 236

ISBN : 9782713223273

ISSN : 0335-5985

\section{Référence électronique}

Daniel Vidal, « Alain Mothu, Gianluca Mori (textes réunis par), Philosophes sans Dieu.Textes athées clandestins du xvıII siècle ", Archives de sciences sociales des religions [En ligne], 156 | octobredécembre 2011, document 156-84, mis en ligne le 16 février 2012, consulté le 21 septembre 2020. URL : http://journals.openedition.org/assr/22930 ; DOI : https://doi.org/10.4000/assr.22930

Ce document a été généré automatiquement le 21 septembre 2020.

(c) Archives de sciences sociales des religions 


\title{
Alain Mothu, Gianluca Mori (textes réunis par), Philosophes sans Dieu.Textes athées clandestins du $\mathrm{XVIII}^{\mathrm{e}}$ siècle
}

\author{
Paris, Ếditions Honoré Champion-Classiques, [2005] 2010, 400 p.
}

Daniel Vidal

\section{RÉFÉRENCE}

Alain Mотнu, Gianluca MoRI (textes réunis par), Philosophes sans Dieu.Textes athées clandestins du XVIII siècle, Paris, Ếditions Honoré Champion-Classiques, [2005] 2010, $400 \mathrm{p}$.

1 L'athéisme, en sa conception la plus radicale, pouvait-il être " pensé » et pensable, au $\mathrm{XVI}^{\mathrm{e}}$, comme accomplissement de la raison même? L'incroyance s'identifiait-elle alors avec l'athéisme? Dans La religion de Rabelais, Lucien Febvre, au milieu du $\mathrm{xx}^{\mathrm{e}}$ siècle, observait que l'on pouvait «se dire, se croire, être chrétien, et vouloir, avant tout, libérer les fidèles, les simples croyants, de terreurs enfantines et de superstitions grossières ». Le siècle s'en prit aux dogmes et aux institutions, mais ne fut pas un " siècle sceptique, un siècle libertin, un siècle rationaliste ". Le glorifier comme tel eut été «la pire des erreurs». Si l'athéisme trouve au xviII ${ }^{\mathrm{e}}$ siècle sa formule exacte et la toute-puissance de sa conviction, c'est sans doute qu'il rompt avec l'invasion mystique du XvII ${ }^{\mathrm{e}}$, ce « siècle des saints » qu'il est convenu, depuis Bremond, d'interroger comme tel. Il serait au demeurant passionnant de tenter de comprendre s'il n'y eut pas, dans cet excès de mysticisme, quelque argument capable de faire basculer l'extrême religiosité en sa négation pure, l'athéisme... Quoi qu'il en soit, le xvIII siècle fut bien, de ce point de vue, le siècle des Lumières où la question de Dieu en tant que telle fut posée avec le plus de rigueur. Du Marsais, D'Holbach, Diderot, les Encyclopédistes, ont au 
cours du siècle fondé l'athéisme comme expérience, et exigence ultime, de la raison. Leurs œuvres sont publiées, accessibles, lumineuses. D'autres philosophes ou "penseurs libres", aujourd'hui dûment identifiés, ou demeurés anonymes, ont laissé, tout au long du siècle (années 1700-1730, puis dans la décennie 1760), des textes ici rassemblés par A. Mothu et G. Mori, qui les affranchissent de leur clandestinité. Ce sont ces textes qui annoncent et fécondent les ouvrages contemporains ou ultérieurs des "grands maîtres". Curieusement, parmi ceux-ci, un auteur oublié de façon incompréhensible par nos deux présentateurs : La Mettrie, son Histoire naturelle de l'âme (1745) et son Homme machine (1747). Oubli délibéré ? Diderot lui avait reproché la " dérive » immoraliste de son système matérialiste, mais celui-ci parait tout de même en parfaite résonance avec celui des plus grands de ses contemporains. Par contre, et à juste titre, l'on peut référer sans difficulté ces textes athées aux philosophes anglais, Locke, Hobbes, Toland qui, les premiers, "ont bâti les fondements successifs d'une pensée intégralement rationnelle », selon l'analyse d'Alain Sandrier, présentant De la raison, texte « clandestin » du baron d'Holbach (fin années 1760). Ce document est pour l'essentiel la traduction par le baron de l'ouvrage majeur de Toland (1670-1722), Christianity not Mysterious (1696), où la raison est promue comme seul guide adéquat à l'interprétation, et tout mystère dès lors réfuté comme pur produit de l'imagination, cette folle du logis.

2 Ces textes réhabilités ne forment pas un ensemble homogène, et ne proviennent pas d'une école spécifique de pensée. Ils n'en prennent que plus d'importance et de force dans la constitution de l'athéisme, ce spectre qui ne cessera plus de hanter, en Occident, philosophes et hommes de simple bon sens. Une constellation de principes en organise cependant l'unité, sans qu'elle s'institue en doctrine ni, véritablement, système. Parmi ces principes, ceux-ci, relevés par les préfaciers : le refus argumenté de l'existence d'une cause intelligente à l'« origine » de l'univers; la défiance à l'égard de toute transposition du sacré au plan de la nature, conçue comme triomphe du «bel ordre »; l'âme, propriété du corps humain ; un rationalisme déterministe qui conduit les auteurs à récuser tout libre arbitre. De Bayle, en amont, à Voltaire, en fin de siècle, la gamme des références et inférences ne cessera de se décliner en chacun de ces textes clandestins selon la pente singulière de leur pensée, et de leurs enjeux.

3 L'oratorien Du Marsais (1676-1756) forge une conception du monde « où Dieu est absent ». Le manuscrit Le Philosophe, (années 1716-1720) pose que "nul être suprême n'exige de culte des hommes ", et que Dieu n'est qu'un "mauvais usage de la faculté de penser ». Sans doute devrait-on repérer en ses travaux approfondis sur le langage et son style (Les Tropes, 1730), l'assise de son argumentation. Son plaidoyer pour ce que l'on a pu appeler « la transparence des signes » et « l'éthique de l'écriture » fond à mon sens son athéisme premier. Ainsi de la proposition selon laquelle « le philosophe prend la maxime à sa source, en examine l'origine, et n'en fait que l'usage qui lui convient ». Certes, la source de nos connaissances « est entièrement hors de nous » - et Du Marsais récuse ainsi le protocole de la méditation, et se réfère à Locke et à sa conception du savoir humain comme relevant des seules « connaissances sensibles »- mais élaborées par la pensée, qui est "en l'homme un sens comme la vue et l'ouïe». Point de transcendance, ainsi, mais point "d'exil du monde». Tout le contraire: "Notre philosophe ne croit point être en pays ennemi, il veut agir en sage économe des biens que la nature lui offre", et avoir commerce avec autrui selon les impératifs de "son unique religion: l'honneur et la probité ». Il est alors remarquable que l'exigence 
d'athéisme implique ici, et comme sa ressource naturelle et sa conclusion nécessaire, le paradigme de l'altérité. Il n'est de sujet authentique, pourrait-on dire, qu'en l'absence de Dieu et la présence des hommes. Du Marsais : «La société civile est, pour ainsi dire, la seule divinité qu'il [le philosophe] reconnaisse sur la terre ». Aussi bien les préfaciers n'ont-ils pas manqué de souligner la "modernité» de ce texte clandestin, "qui considère comme accompli le procès de sécularisation à peine entamé ». Tout se joue ainsi en un seul mouvement de la pensée, qui ne décide de la possibilité de l'homme «civil» que sous condition de la négation de tout dieu, et du concept de société que sous condition de désenchantement.

4 Dans son traité sur "L'origine des êtres et des espèces ", rédigé dans les années 1705-1710, Henri de Boulainviller (1658-1722) en appelle plus à un matérialisme athée, joignant cartésianisme et mécanicisme, qu'à des considérations "anthropologiques ». Et la question de l'infinité de l'univers se pose alors en toute rigueur. Boulainviller prône une conception strictement déterministe et évolutionniste, «infinitiste », de la matière, «ce fluide en mouvement, hétérogène ", porteur de tous les possibles, pures virtualités ou réalisations avérées : «Tout ce qui est dit possible doit être conçu comme existant, ou ayant existé, ou devant exister ». Le temps ne peut se concevoir que sous la catégorie de l'éternité, mais en un déploiement infiniment continu et progressif: «Dans la supposition du mouvement, le laps du temps équivaut à l'intelligence » - ce que l'on tient pour « intelligence » n'est que l'autre nom du déploiement naturel de la matière. Et, au-delà de cette considération, qui supprime le recours à toute causalité intelligente extérieure à ce mouvement naturel, la pensée athée s'ouvre pleinement à l'accueil de l'improbable: "Il n'y a nulle combinaison qui ne puisse arriver dans un certain temps, et, partant, dans l'éternité ». Le lecteur pourrait à bon droit invoquer ici l'utopie fouriériste et ses improbables germinations de chimères. Si les préfaciers n'en viennent pas à telle comparaison, ils n'en concluent pas moins que, pour Boulainviller, " une matière infinie devra assumer tôt ou tard toutes les configurations qui ne sont pas contradictoires avec son essence». Ainsi se dénonce tout spiritualisme, et toute évocation d'un libre arbitre. Car la matière en tous ses états est par "essence " l'univers infiniment déterminé.

5 Ce n'est que vingt ans après sa mort que sera publiée, en 1768, la Lettre de Thrasybule à Leucippe, rédigée dès les années 1720-1725 par Nicolas Fréret (1688-1749). Fréret est juriste, historien encyclopédique, précepteur chez le duc de Noailles, etc. - homme de grand talent et de bel entourage. "Penseur libre», écrivent les préfaciers, dans la mouvance et la distance de Descartes, Malebranche, Locke. À la base de son athéisme, la faillite des religions révélées: le seul critère de validité des connaissances est l'expérience. Sa théorie des " distinctions » le conduit à privilégier l'existence "réelle » des choses par rapport à leur existence « objective ». Objective, c'est-à-dire abstraite, considérée comme entité réifiée. Ainsi de Dieu, qui répond à la question de la cause universelle et intelligente, et qui, dans l'usage des mots qui le disent, échappe à la "réalité» pour devenir pure fiction. Il n'est point, chez Fréret, d'appel à transcendance, non plus qu'à immanence. Dieu n'est pas l'autre nom de la Nature, et le juriste rejoint ici très précisément Pierre Bayle dans son opposition à la conception spinoziste. " "Divinité", "destinée", "providence", fantômes auxquels on attribue une réalité que n'a jamais eue l'image confuse qui les accompagne». Tout ce qui se dit oracles, révélations, tout ce qui métamorphose un homme en roi et un roi en un dieu, tout ce qui se donne comme livre sacré, ne sont que preuves fallacieuses de l'existence de Dieu, car non fondées « sur les lumières de la raison naturelle, de la vraie raison (...) 
On en est venu enfin à imaginer qu'il pouvait exister une Cause qui ne fût pas un être ou un corps ».

6 Mais il est aussi chez Fréret une dimension anthropologique, qui fonde l'athéisme sur l'alternative du plaisir et de la douleur. Non pas dubien et du mal, qui pourrait réintroduire la question de Dieu par la quête du salut. Chercher son plaisir ou fuir la douleur, telle est la seule question véritable que se pose l'existence humaine. Par l'exemple de Zaradès le Persan (Zoroastre), Fréret veut prouver que «de toutes les religions qui nous sont connues, c'est la plus sensée », qui implique de « rechercher le bien dans le cadre de l'ordre social». Mais «après tout, poursuit Fréret, son instaurateur n'est qu'un simple homme qui ne nous prouve point qu'il ait d'autre droit que celui de la raison ». Coup double : telle " religion » est un fait d'homme ; la quête du bien, et son accomplissement, ne s'entendent que sous condition de vie sociale. Du Marsais avait sollicité la "société civile " comme seule dépositaire légitime, et en dernière instance, $d u$ «sacré ». En invoquant la recherche du bonheur comme instituant de cette société, Fréret libère l'athéisme de ce qui pouvait apparaître, tout anachronisme assumé, comme possible antihumanisme. C'est de l'homme que provient Dieu, par agrégations de réponses confuses à des questions sans issues, de qualités purement humaines et d'un seul coup de force sacralisées. "On joint ensemble des idées de cause, d'intelligences, de volontés, de puissance, de bonté ou de malice, et l'on donne le nom de Dieu à cet assemblage. On s'accoutume à le considérer comme quelque chose de réel, et on oublie que c'est son propre ouvrage ». Dieu n'« est » donc pas, sinon comme «fantôme». Mais qu'est-ce alors qui en «tient lieu»? Rien. Fréret: "Je supporte sans douleur le vide que les théistes croient remplir par la supposition d'une Cause intelligente »: contre le créationnisme auquel Fréret s'oppose de toutes ses forces, et que l'on appellerait aujourd'hui « Dessein Intelligent », cette Lettre longtemps clandestine aujourd'hui peut se lire en grande jubilation: dans l'univers, «je ne découvre de bornes ni par son étendue ni par sa durée; j'y aperçois seulement une vicissitude et un passage continuel d'un état à l' autre, (...) mais je n'y vois pas une Cause universelle».

7 "Lecteur avide de Bayle", l'avocat André-Robert Perrelle (1695-1731), dans son manuscrit Sur les preuves de l'existence de Dieu, rédigé dans les années 1715-1720, partage la conception évolutionniste de Fréret, et son anti-créationnisme, "car il lui est impossible, rappellent les présentateurs, de concevoir une étendue/matière non existante ». La matière est cela qui est infini, et de toute éternité. L'univers, cet amas de "poussière divisée à l'infini » se déploie en tourbillons, qui s'organisent selon le rapport nécessaire entre les choses, qui est leur propre loi. "Qu'est-il besoin d'intelligence? Le seul mouvement suffit pour produire tous les effets que nous admirons tous dans la nature ». Le mouvement, qui est la loi de la matière. Son principe d'existence, tout Dieu récusé. L'influence de Toland est ici manifeste. De même que dans le texte qu'un philosophe, demeuré anonyme, rédige fin 1720. L'auteur des Essais sur la recherche de la vérité participe aux "cercles de l'incroyance parisienne ", assurés de la protection d'éminentes personnalités, dont Maurepas. L'athéisme est ainsi de versant hauturier, et cela ne doit pas surprendre: en ces cercles se débattaient les philosophies et les conceptions du monde, à l'écoute des grandes voix d'Angleterre ou des Provinces-Unies, qui libéraient peu à peu les chemins de plus grande aventure. Ainsi pour Étienne Guillaume, curé de Fresnes, dont le traité sur L'âme matérielle (1728) s'élève contre la substantialité de l'âme, et le libre-arbitre. Les Essais refusent aussi bien tout innéisme et tout spiritualisme. Et, comme les textes précédents, critiquent le 
postulat d'une « cause première, immatérielle, intelligente de l'univers ». On ne recourt à telle explication que sous la contrainte des passions et des angoisses quant à l'origine et au destin du monde. De là que les conclusions soient viciées : «Ce n'est que dans les choses absolument détachées de tous nos intérêts et de toutes nos passions que nous découvrons la vérité ». Ainsi en va-t-il de la géométrie, - ainsi doit-il en aller de nos considérations cosmologiques ou anthropologiques. «Il n'y a dans la disposition de l'univers aucunes vues particulières ou tendantes à pourvoir aux besoins des hommes (...) Le premier arrangement [de l'univers] est d'une telle simplicité que ce n'est point trop donner au hasard que de l'en croire le principe». Nulle "providence», nul « dessein particulier» ne peuvent être convoqués au grand jeu de hasard d'un univers de pure matérialité. Et si les passions émanent des « principes corporels ", l'âme, qui en est le « siège ", dépérit quand « le corps devient languissant et approche de sa fin ».

D'un premier tiers de siècle à ses dernières décennies, les mêmes conceptions s'affirment et se reprennent. Que «l'idée de Dieu » soit « un préjugé enraciné dans l'esprit de tous les hommes ", cela vaut destitution de tout appel à divinité, car préjugé s'oppose à vérité et à raison. L'auteur des Réflexions sur l'existence de l'âme et sur l'existence de Dieu, écrit avant 1734, publié en 1743, pose l'examen comme condition de l'accès à la vérité : "On s'est fait une habitude de croire sans examiner »- et examen, ici, se dit de cette opération de la raison qui discrimine en toutes choses et pensées le réel de l'imaginé. Et c'est dans l'intimité de sa propre "conscience " que cette opération se décide : «De tous les êtres qui existent, aucun n'a un rapport plus intime avec l'homme que l'homme même ». C'est de cet homme, sans doute, que procède Dieu ; mais c'est cet homme qui en dit l'impossible nécessité, et son impensable " existence ". Vers la fin des années 1760, un traité contemporain des écrits de d'Holbach et Diderot rassemble une nouvelle fois les réquisits de l'athéisme, et ses impératifs. Le Jordanus Brunus redivivus redonne la priorité à l'expérience, à la matérialité seule de l'univers, à la nécessité de faire lumière sur toutes choses, car «le grand jour est trop fatal à tout système de religion ", qui n'a pour seule défense que le recours à la persécution, dont G. Bruno est la figure emblématique du martyre. Le philosophe anonyme est, de tous les auteurs présentés, le seul qui porte explicitement le fer contre les prêtres et leurs "grandes fureurs». Toute "démonstration physique» les "excite», et la position matérialiste radicale et infinitiste leur est irrecevable, lorsqu'elle justifie l'hypothèse de la pluralité des mondes : car «il n'y a point, écrit l'auteur, de répugnance de la part de la matière, qui se peut accroître infiniment ». Si l'on admet un Dieu, «il se présente un grand nombre d'impossibilités que tout l'art des sophistes ne saurait détruire». Par exemple, la possibilité du mal, incompatible avec l'existence de Dieu, de même que la conception d'une matière sans commencement ni fin, qui rend l'hypothèse d'un Dieu créateur, « conservateur et rémunérateur, (...) chose absolument inutile».

9 Ainsi s'écroule, aux yeux des philosophes athées, tout l'édifice des croyances en Dieu, et l'institution même de religion. Dans le texte du baron d'Holbach, De la raison (fin des années 1760), que nous savons pour l'essentiel repris de Toland, le philosophe note que cet ensemble de critiques, fondé sur la seule raison et la seule expérience des sens, l'une n'allant jamais sans l'autre, "nous jette dans le scepticisme et même dans l'athéisme absolu ». La révélation, support de la religion " heurte la conviction, écrit A. Sandrier en sa présentation, bafouant l'universalité des principes rationnels ». Il en est fini du « siècle des saints » évoqué plus haut. Place est désormais nette pour que les 
Lumières, en la négation argumentée de Dieu, disposent tout savoir dans le vertige redoutable d'une liberté sans autre limite que sa propre déraison. 